\title{
Acute Psychosocial Stress Reduces Cell Survival in Adult Hippocampal Neurogenesis without Altering Proliferation
}

\author{
Rosanne M. Thomas, Gregory Hotsenpiller, and Daniel A. Peterson \\ Neural Repair and Neurogenesis Laboratory, Department of Neuroscience, The Chicago Medical School at Rosalind Franklin University of Medicine and \\ Science, North Chicago, Illinois 60064
}

\begin{abstract}
Factors modulating neurogenesis may contribute to the pathophysiology of affective disorders such as major depression. Environmental stressors in animal models have been proposed to alter neurogenesis, suggesting a mechanism for this contribution. The effect of an acute psychosocial stressor on either proliferation or survival (immediate, short term, and long term) was examined along with subsequent neuronal differentiation in the hippocampus of adult male Sprague Dawley rats. Subjects were exposed to a widely used social dominance paradigm that elicits behavioral and physiological responses to an acute psychosocial stressor. This social dominance paradigm may mimic human relational stress more realistically than laboratory stressors and provides a socially relevant model. We found that exposure to an acute psychosocial stressor at the time of cell generation resulted in a decreased number of newly generated cells in the hippocampus. By using sequential thymidine analog administration to provide temporal discrimination of DNA replication, we showed that short-term survival but not initial proliferation or immediate survival was altered in response to stress. Furthermore, we determined that stress experienced subsequent to proliferation also diminished long-term survival of cells. Thus, an acute episode of a social stress produces long-lasting effects on the incorporation of new hippocampal neurons by reducing their survival.
\end{abstract}

Key words: depression; dentate gyrus; cell cycle; confocal stereology; IdU; CldU

\section{Introduction}

The experience of stress in humans has been implicated as a crucial component in the emergence of affective disorders such as depression (Kessler, 1997; Jacobs et al., 2000a,b; McEwen, 2000). Stress has been shown to lead to diminished neurogenesis in primates in a laboratory setting (Gould et al., 1997, 1998; Fuchs et al., 2001), and stress-induced reduction in neurogenesis has been proposed as a contributor to the pathophysiology of depression (Fuchs and Flugge, 1998; Gould and Tanapat, 1999; Czeh et al., 2001; Gould and Gross, 2002). An association between neurogenesis and depression is supported by the fact that both are regulated by antidepressants (Duman et al., 1999, 2001a,b; Czeh et al., 2001; Malberg, 2004). Stress-induced reduction of neurogenesis in humans with unipolar major depression has been suggested as a basis for observed hippocampal volume reduction in these patients (Sheline, 2000).

Stress produced through social interactions is ubiquitous across mammalian species. Therefore, an animal model of psychosocial stress may be relevant for investigating a link between modulation of neurogenesis and depression. Natural stressors, such as social defeat, are more salient to humans than typical

Received Sept. 5, 2006; revised Jan. 10, 2007; accepted Jan. 29, 2007.

This work was supported in part by National Institutes of Health Grant AG20047 to D.A.P. We thank Drs. Janice Urban and Tara Teppen for assistance with the CORT analysis and Dr. Sarah Garber for a critical reading of this manuscript.

Correspondence should be addressed to Daniel A. Peterson, Neural Repair and Neurogenesis Laboratory, Department of Neuroscience, The Chicago Medical School at Rosalind Franklin University of Medicine and Science, 3333 Green Bay Road, North Chicago, IL 60064. E-mail: daniel.peterson@rosalindfranklin.edu.

D01:10.1523/JNEUROSCI.3849-06.2007

Copyright $\odot 2007$ Society for Neuroscience $\quad$ 0270-6474/07/272734-10\$15.00/0 laboratory stressors (such as footshock) and afford broader relevance of animal study outcomes to humans. Psychosocial stress produces robust, measurable behavioral changes in animals analogous to behavior exhibited in depressed humans, and it closely mimics the onset of stress-related pathology in humans (Kessler, 1997; Blanchard et al., 2001). Chronic stress decreases adult neurogenesis in the hippocampus (Fuchs and Flugge, 1998; Gould et al., 1998; Czeh et al., 2001, 2002; Fuchs et al., 2001; Malberg and Duman, 2003; Pham et al., 2003); however, little is known about the effect of an acute episode of naturally occurring stress on neurogenesis, although it is reported to diminish neuronal proliferation in tree shrews (Gould et al., 1997). It is not known whether an acute episode of psychosocial stress could have an effect on the proliferation, differentiation, or long-term survival of newly generated neurons in hippocampal neurogenesis or whether this alteration of neurogenesis could contribute to depression.

One barrier to understanding the potential regulation of neurogenesis by stress has been a lack of precision in defining what stage of the process is vulnerable. Evidence is accumulating that neurogenesis is a multistep process (Gage et al., 1995; Kornack and Rakic, 1999; Gould et al., 2000; Malberg and Duman, 2003; Garcia et al., 2004; Malberg, 2004; Encinas et al., 2006). Temporal studies of phenotypic markers of lineage commitment suggest that there are at least six sequentially distinct stages of neurogenesis after the generation of a neuronal progenitor cell (Kempermann et al. 2004). It is likely that the regulatory mechanisms involved may differ between stages of neurogenesis. To investigate which temporal stage of neurogenesis may be susceptible to alteration by acute psychosocial stress, we designed a study of 
neurogenesis to separately evaluate the alteration of proliferation, immediate survival, or short-term survival of newly generated cells or the differentiation and long-term survival of new neurons in the hippocampus.

\section{Materials and Methods}

To determine whether acute stress differentially modulated the different stages of neurogenesis, we undertook a study designed to discriminate between sequential events of neurogenesis to ascertain which stages were altered by stressor exposure. Specifically, the experimental design discriminated between the effect of an acute psychosocial stressor on proliferation, immediate survival, short-term survival, and neuronal differentiation-long-term survival of newly generated cells in the hippocampus. A social dominance paradigm widely used in behavioral studies was used with adult male Sprague Dawley rats (Hotsenpiller and Williams, 1996; Blanchard et al., 2001). Precise timing of thymidine analog delivery relative to the stress episode allowed for an analysis of specific neurogenic phases (Vega and Peterson, 2005).

This study assessed the effect of an acute psychosocial stressor on four separate phases of neurogenesis: (1) proliferation, (2) immediate survival, (3) short-term survival, and (4) differentiation-long-term survival in the hippocampal dentate gyrus of adult rats (see Fig. 1). Psychosocialinduced stress was measured by evaluating animal behavior and plasma corticosterone (CORT) levels relative to control animals. Qualitative analyses of newly generated cells were performed with immunoperoxidase and immunofluorescence immunostaining to determine cell distribution and morphology. Design-based stereology was used to estimate the total number of newly generated cells. Experiment 1 (see Fig. $1 B$ ) assessed the effects of stress on the early events in neurogenesis, namely proliferation and immediate cell survival ( $24-48 \mathrm{~h}$ ). Experiment 2 (see Fig. $1 C$ ) investigated the acute stress effect on short-term cell survival (1 week). Experiment 3 (see Fig. 1D) investigated the effect of acute psychosocial stress on differentiation and long-term survival of newly generated cells ( 4 weeks). Thus two populations of cells were investigated: (1) cells generated concurrent with stress (experiments 1 and 2) and (2) cells generated before the stress episode (experiment 3 ). The results of each experimental group are not directly comparable because stress and thymidine analog administration time frames differ; however, each of the three experiments provides complementary data.

\section{Animal subjects}

Resident colony animals. To generate the paradigm of psychosocial stress, six resident colonies were established, each consisting of two male Sprague Dawley rats, 9-12 months of age (weighing 450-600 g), hereafter termed resident rats, and a female Sprague Dawley rat, 6-9 weeks of age. Resident rats were housed in polypropylene tubs $(50 \times 40 \times 20 \mathrm{~cm})$ that were slightly larger than standard group-housing cages and were on a reversed $12 \mathrm{~h}$ light/dark cycle. The light/dark cycle was reversed for resident rats only to ensure increased activity of resident rats during a standardized morning testing time of 10:00 A.M. Corn cob bedding material covered the tub floor and was changed every week, but not before defeat sessions, because the presence of odors enhances the aggressiveness of rat colonies (Williams et al., 1990; Hotsenpiller and Williams, 1996).

Intruder animals. Naive adult male Sprague Dawley rats, 6-9 weeks of age (weighing 200-250 g), were housed in groups of three. These naive rats, hereafter called intruder rats, were introduced individually into established resident rat colonies, as described below. All rats in all conditions had access to food and water ad libitum and a $12 \mathrm{~h}$ light/dark cycle. Testing occurred during the intruder rat light part of the standard $12 \mathrm{~h}$ cycle (the dark cycle for resident rats). All animal procedures were performed in accordance with National Institutes of Health guidelines and were approved by the Institutional Animal Care and Use Committee of Rosalind Franklin University of Medicine and Science.

\section{Behavioral model: social dominance paradigm}

The social dominance paradigm was modified from the protocol of Hotsenpiller and Williams (1996). Six colonies were established consisting of two aggressive resident rats housed with a young female to increase res- ident rat aggression and territorial behavior. Colonies were trained with the introduction of a naive non-subject training intruder rat three times over a period of 1 month before subject testing. Colonies were selected for inclusion in the study based on resident rats fulfilling the aggression criteria of consistent biting and pinning down of non-subject training intruder rats within the first $5 \mathrm{~min}$ of their introduction. For testing, the female was removed and a naive young male Sprague Dawley subject, designated as the intruder, was placed in the colony for $20 \mathrm{~min}$. Intruder rats were typically pinned down and/or bitten by the dominant rats, creating an acute psychosocial stress (see Fig. 2). Criteria for acceptance of an intruder subject into the study included submissive posture and being pinned down by the dominant resident rat within 1 min after introduction into the colony cage. All intruder subjects met these criteria. Bites occasionally required treatment, including the application of antiseptic ointment and sutures, but none of the subjects became physically impaired as a result of the stressful encounters. To control for nonspecific stress, control rats were removed from their group home cages and placed alone in a new room and a new non-colony, standard-size cage with fresh bedding for $20 \mathrm{~min}$.

\section{Determination of CORT levels}

CORT levels are a common measure of an animal's state of stress. Blood samples for CORT determination were obtained from experiment 2 (short-term survival) intruder $(n=5)$ and control $(n=3)$ rats before return to their home cages. The process of tail clip and blood collection took $<2 \mathrm{~min}$ for each rat. The tail clip was performed on the distal 0.5 $\mathrm{mm}$ of the tail; $0.5 \mathrm{cc}$ of blood was collected in a small heparinized vial; the tail was sprayed with antiseptic spray; and the animal was returned to its home cage (Meerlo et al., 2002). Blood was kept on ice briefly until centrifugation $\left(1500 \mathrm{rpm}\right.$ at $-20^{\circ} \mathrm{C}$ for $15 \mathrm{~min}$ ) to allow for extraction of plasma. Plasma was kept at $-80^{\circ} \mathrm{C}$ until analysis. CORT levels were determined by using the Immuno-Chem Double Antibody Corticosterone- ${ }^{3} \mathrm{H}$ Kit for rats and mice (MP Biomedicals, Irvine, CA).

\section{Thymidine analog labeling}

Control and intruder subjects received injections of one or more thymidine analogs at $12 \mathrm{~h}$ intervals for $3 \mathrm{~d}$ to label cycling cells. Injections labeled a cohort of cells that were undergoing mitotic S-phase at the time the label was present. Up to $3 \mathrm{~d}$ of thymidine analog injections at $12 \mathrm{~h}$ intervals were given to ensure that there was an adequate population to detect statistically testable changes in cell numbers with stress. Experiments were designed to present psychosocial stress at different times of neurogenesis relative to the initial events of DNA replication (see Fig. 1). Thymidine analog availability was timed to coincide with experimental stress to measure the effect on cell proliferation and immediate and short-term survival (experiments 1 and 2) (see Fig. $1 B, C$ ). Animals in experiments 1 and 2 received their last dose of thymidine analog administration just before their psychosocial stress encounter. Delaying the stress paradigm to 1 week after thymidine analog injection allowed us to examine the effect of the psychosocial stress event on long-term survival and differentiation of newly generated cells (experiment 3) (see Fig. 1D).

Chlorodeoxyuridine and iododeoxyuridine labeling. To sequentially assess stress effects on proliferation and immediate survival, experiment 1 subjects (see Fig. $1 B$ ) (control, $n=4$; intruder, $n=4$ ) received intraperitoneal injections of iododeoxyuridine (IdU) $(57.5 \mathrm{mg} / \mathrm{kg}$; MP Biomedicals) at $12 \mathrm{~h}$ intervals for $2 \mathrm{~d}$ followed by $1 \mathrm{~d}$ of chlorodeoxyuridine (CldU) (42.5 mg/kg; Sigma, St. Louis, MO) intraperitoneal injections at a $12 \mathrm{~h}$ interval to label cycling cells. Equimolar administration ensured the equal availability of analog for quantitative comparison (Vega and Peterson, 2005). Briefly, solutions of IdU and CldU were prepared to achieve the same molar concentration as bromodeoxyuridine (BrdU) (equimolar delivery at $65 \mathrm{~mm}$ ). Equimolar solutions for IdU were 23 $\mathrm{mg} / \mathrm{ml}$; for CldU, they were $17 \mathrm{mg} / \mathrm{ml}$. Therefore, injections at $2.5 \mathrm{ml} / \mathrm{kg}$ resulted in an equimolar final delivery of $57.5 \mathrm{mg} / \mathrm{kg}$ IdU and $42.5 \mathrm{mg} / \mathrm{kg}$ CldU to be equivalent to a BrdU administration of $50 \mathrm{mg} / \mathrm{kg}$, as used in all BrdU injection protocols throughout this study. Subsequent detection of CldU revealed those cells undergoing DNA replication (proliferation) at the time of the stress episode, whereas detection of IdU revealed the cell population generated immediately before stress exposure, providing for 


\section{A. Sequence of Neurogenesis}

\begin{tabular}{c|c|c|c|c|}
\multicolumn{1}{c|}{ Immediate Short Term Term Survival } \\
\hline Proliferation & Lineage Commitment
\end{tabular}

\section{B. Experiment 1: Assessment of Proliferation (CldU) and} Immediate Survival (IdU)

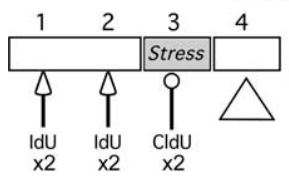

C. Experiment 2: Assessment of Short Term Survival

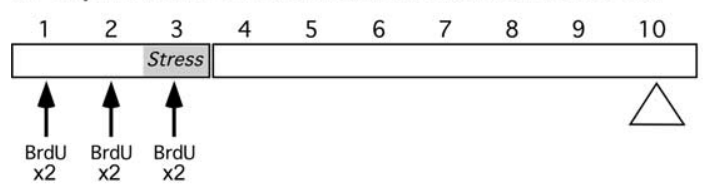

\section{Experiment 3: Assessment of Long Term Survival}

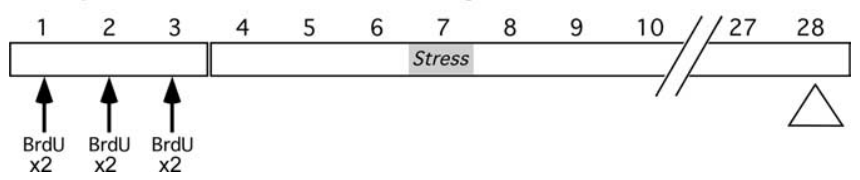

Figure 1. Design of the study. $\boldsymbol{A}$, Adult neurogenesis in the hippocampal dentate gyrus consists of a process beginning with DNA replication during proliferation of uncommitted progenitor-stem cells that subsequently undergo lineage commitment. Not all newly generated cells survive, and it is the combination of survival and lineage commitment that determines the extent to which new neurons are added to the hippocampus. This study evaluated the modulation of stress on several stages of this continuum of neurogenesis. $\boldsymbol{B}$, To discriminate the effect of stress on cellular proliferation and immediate survival, two different thymidine analogs, IdU and CIdU, were sequentially delivered because they can be separately detected by immunohistochemistry. IdU was administered first to provide a measure of immediate survival, and the episode of acute stress coincided with administration of CIdU to evaluate stress modulation of proliferation when assessed the next day (day 4). C, To assess the stress effect on short-term survival, a cohort of proliferating cells was identified by incorporation of another thymidine analog, BrdU, coincident with an episode of acute stress, and evaluated for short-term survival after 1 week (day 10). D, To further discriminate between proliferation and survival, a cohort of newly generated cells was identified by BrdU administration and subjected to acute stress 1 week after birth and evaluated for long-term survival after 4 weeks (day 28). Arrows indicate thymidine analog delivery (two times daily); triangles indicate time of tissue collection.

assessment of immediate cell survival (see Fig. $1 B$ ). Reentry into an additional cell cycle was revealed by cells incorporating both CldU and IdU. On day 4, experiment 1 subjects were anesthetized deeply and transcardially perfused ( $5 \mathrm{~min}$ with ice-cold saline followed by ice-cold $4 \%$ paraformaldehyde in $0.1 \mathrm{~m}$ phosphate buffer for $10 \mathrm{~min}$ ).

BrdU labeling. To evaluate stress effects on the early events in neurogenesis, i.e., short-term cell survival, experiment 2 subjects (see Fig. 1C) (control, $n=3$; intruder, $n=5$ ) received $3 \mathrm{~d}$ of BrdU delivery $(50 \mathrm{mg} / \mathrm{kg}$, i.p.; Sigma, St. Louis, MO) at $12 \mathrm{~h}$ intervals (and then were exposed to the social dominance stress on day 3 . On day 10 , experiment 2 subjects were anesthetized deeply and transcardially perfused for $5 \mathrm{~min}$ with ice-cold saline followed by ice-cold $4 \%$ paraformaldehyde in $0.1 \mathrm{~m}$ phosphate buffer for $10 \mathrm{~min}$.

To further elucidate the effect of stress on the long-term survival and differentiation of newly generated cells, experiment 3 subjects (see Fig. $1 D$ ) (control, $n=6$; intruder, $n=9$ ) received BrdU delivery at $12 \mathrm{~h}$ intervals on days 1,2, and 3 and did not experience the social dominance stress until day 7 . This interval was sufficient to allow newly generated cells to undergo differentiation within the hippocampus and thus enable us to measure stress effects on differentiation-long-term survival separate from cell generation (Fig. 1D). Perfusion occurred on day 28 (3 weeks after the stress exposure).

Immunohistochemistry. After perfusion, brains were removed, placed in fixative solution overnight, and then equilibrated with 30\% sucrose. Serial, $50-\mu \mathrm{m}$-thick sagittal sections were cut by freezing microtomy and stored in cryoprotectant. A one-in-six series of sections was taken for analysis. Before immunostaining, sections were pretreated to enable nuclear thymidine analog staining, as described previously (Gage et al., 1995). Briefly, sections were rinsed three times in Tris-buffered saline (TBS), incubated free-floating in formamide/SSC solution in a water bath at $65^{\circ} \mathrm{C}$ for $2 \mathrm{~h}$, rinsed in $2 \times \mathrm{SSC}$ solution, incubated in $2 \mathrm{~N} \mathrm{HCl}$ in a water bath at $37^{\circ} \mathrm{C}$ for $30 \mathrm{~min}$, and then rinsed in $0.1 \mathrm{M}$ borate buffer. Six final rinses were done in TBS before further tissue processing.

$I d U / C l d U$ detection (experiment 1). Multiple immunofluorescence labeling was used to detect colocalization of IdU and CldU, as described previously (Vega and Peterson, 2005). Lineage markers for cell differentiation were not explored because the time frame of these experiments was too short to expect proliferating cells to have undergone differentiation. Free-floating sections were pretreated as described, rinsed, blocked, and then incubated in pooled primary antibodies for $72 \mathrm{~h}$. Detection of thymidine analogs was accomplished with mouse anti-BrdU (Becton Dickinson Bioscience, San Jose, CA), at a dilution of 1:500 for IdU, and rat anti-BrdU (Accurate Chemicals, Westbury, NY) at a dilution of 1:250 (with a biotinylated secondary antibody for signal amplification) for CldU. Inclusion of antisera against calbindin, a neuronal calcium binding protein (rabbit, 1:1000; Swant, Bellinzona, Switzerland), was used to stain granule neurons to facilitate identification of the granular layer in the dentate gyrus. To determine the phenotypic distribution of IdU- and CldU-labeled cells, another series from each subject was stained with antisera as described above, but the anti-calbindin was replaced with anti-doublecortin (DCX) (goat, 1:250; Santa Cruz Biotechnology, Santa Cruz, CA) to assess the extent of lineage commitment in the proliferating cell population.

All secondary antibodies (Jackson ImmunoResearch, West Grove, PA) were raised in donkey antisera and used at a dilution ratio of 1:500. After subsequent rinsing six times with TBS and blocking two times with donkey antisera, IdU was detected with amplification by using a biotinylated donkey anti-mouse antibody with subsequent exposure to streptavidin conjugated with cyanine 5 (Cy5). CldU was detected with donkey antirat antibody conjugated to Cy2. Calbindin was detected with donkey anti-rabbit secondary conjugated to Cy3. For the series stained with antisera to DCX, the fluorophore assignment was as follows: DCX-Cy2, IdU-Cy3, and CldU-Cy5. Sections were subsequently rinsed, mounted on slides, briefly air dried, and coverslipped with a polyvinyl alcohol/1,4 diazabicyclo [2.2.2] acetane solution for permanent polymerization (Peterson, 2004). The interval between mounting and coverslipping was $<3$ min to prevent tissue shrinkage that occurs with longer exposure to air drying, thus maximizing tissue thickness for stereological quantification (Peterson, 2004).

BrdU detection (experiments 2 and 3). For immunoperoxidase detection of cells incorporating BrdU, sections were pretreated as described above and rinsed repeatedly in TBS followed by a $10 \mathrm{~min}$ incubation with $30 \% \mathrm{H}_{2} \mathrm{O}_{2}$ to inactivate endogenous peroxides. After three additional TBS rinses, sections were blocked with $2.5 \%$ donkey serum and $0.5 \%$ Triton X-100 in TBS for $3 \mathrm{~h}$ and then incubated for $72 \mathrm{~h}$ with anti-rat $\operatorname{BrdU}$ (1:500; Accurate Chemicals) as the primary antibody. After six additional rinses and blocking, sections were incubated with a biotinylated donkey anti-rat secondary antibody (1:500; Jackson ImmunoResearch) for $2 \mathrm{~h}$. Sections were subsequently rinsed and incubated in avidin-biotin solution (ABC/Elite Vectastain kit; Vector Laboratories, Burlingame, CA) for $1 \mathrm{~h}$. Reaction with diaminobenzidine solution was performed for 4 min followed by six TBS rinses. Sections were mounted on slides and air dried overnight. To maximize retention of section thickness for stereological quantitation, sections were placed for $4 \mathrm{~min}$ in a 1:1 solution of chloroform/ethanol, briefly rinsed in an ascending ethanol series, and then briefly rinsed in CitriSolv (Fisher Scientific, Hanover Park, IL) three to five times before being coverslipped in ProTexx (Lerner Laboratories, Pittsburgh, PA).

Multiple immunofluorescence labeling was used to detect colocalization of BrdU with lineage markers for cell differentiation. Free-floating sections, pretreated as described above, were rinsed and then blocked in TBS containing 2.5\% donkey serum and $0.5 \%$ Triton X-100 for $3 \mathrm{~h}$, and 

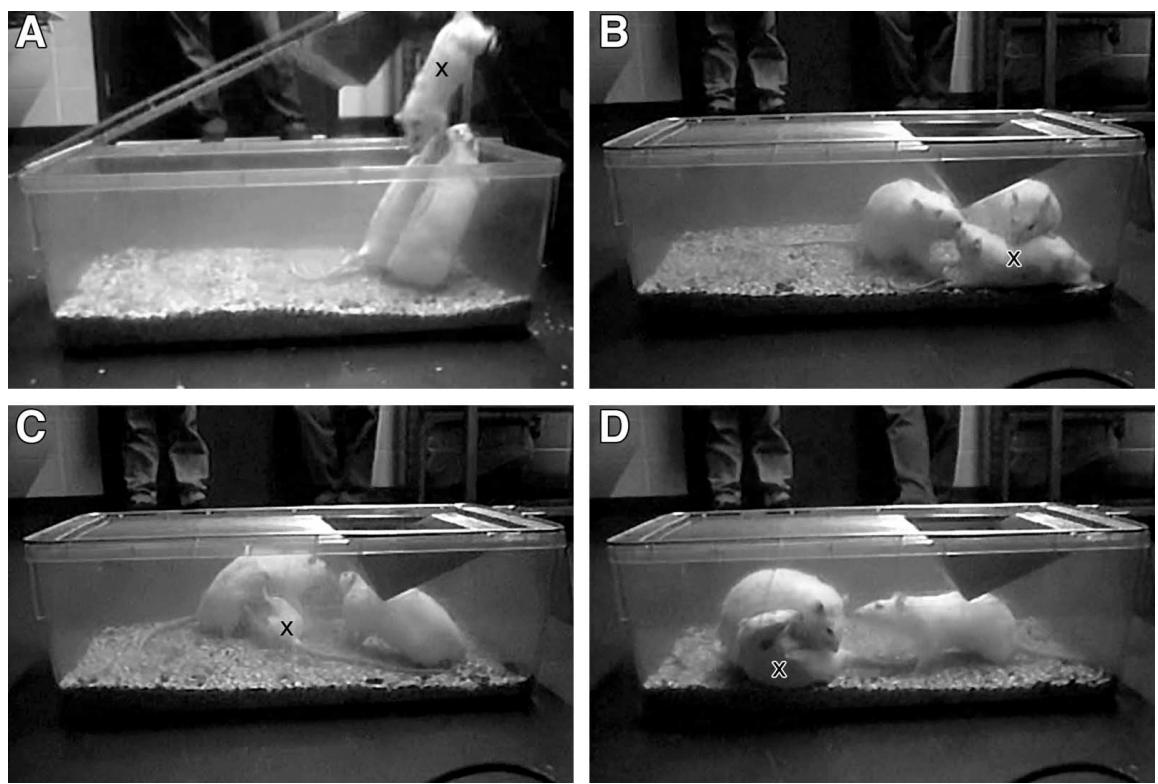

Figure 2. Paradigm for acute psychosocial stress. $\boldsymbol{A}$, A young naive male rat (intruder; marked with the black X) was introduced into a stable colony of two rats whose female companion had just been removed. $\boldsymbol{B}$, During initial exploration, the colony rats became aggressive, and the intruder rat (blackX) exhibited submissive behavior. $\boldsymbol{C}, \boldsymbol{D}$, The aggression continued, with the intruder rat being pinned down and bitten.

then incubated in pooled primary antibodies for $72 \mathrm{~h}$. It was possible to pool all primary antibodies provided they were raised in different host species. Detection of BrdU was accomplished with the anti-rat BrdU described above (1:500; Accurate Chemicals). The lineage markers that we used were mouse anti-neuronal nuclei (NeuN) (1:5000; Millipore, Temecula, CA) for the detection of mature neurons and rabbit antiS100 $\beta$ (1:5000; Swant) for detection of mature astrocyte cell bodies. After subsequent rinsing six times in TBS and blocking two times with donkey serum, BrdU was detected with amplification by using a biotinylated donkey anti-rat antibody with subsequent exposure to streptavidin conjugated with Cy2. Other lineage markers were detected with appropriate secondary antibodies raised in donkey serum and conjugated to Cy3 or Cy5. Sections were mounted, air dried briefly, and then coverslipped in polyvinyl alcohol/1,4 diazabicyclo [2.2.2] acetane as described above.

\section{Stereological quantification}

Proliferation-immediate survival: experiment 1. Detection of IdU and CldU coexpression was accomplished with immunofluorescence-stained tissue. Stereological sampling was performed with confocal laser scanning microscopy (Olympus Fluoview 300) for determination of multiple markers by using $10 \times$ and $60 \times$ [oil; 1.4 numerical aperture (NA)] lenses. The following excitation wavelengths were used: $488 \mathrm{~nm}$ for the secondary fluorophore Cy2 (CldU), $568 \mathrm{~nm}$ for the secondary fluorophore Cy3 (calbindin), and $647 \mathrm{~nm}$ for the secondary fluorophore Cy5 (IdU). The confocal microscope was equipped with a motorized stage and a z-axis motor and interfaced with the StereoInvestigator software (MBF Bioscience, Williston, VT) for stereology.

Estimation of IdU- and CldU-positive cell numbers was performed by using the optical fractionator procedure for confocal stereology as described previously (Peterson, 1999, 2004). IdU- or CldU-positive cells were counted at predetermined intervals $(x=300 \mu \mathrm{m} ; \mathrm{y}=250 \mu \mathrm{m})$ with a counting frame $(250 \times 185 \mu \mathrm{m})$ superimposed on the tissue-section image. Serial focal plane confocal stacks were acquired at each sampling site with a $60 \times$ oil immersion objective (1.4 NA) and a matching confocal aperture for maximal axial resolution. Section thickness was measured directly at each site with the optical dissector counting frame height set at $30 \mu \mathrm{m}$, which is $\sim 70 \%$ of the mounted section thickness with this type of histological processing. This procedure sampled at least $5 \%$ of the total hippocampal dentate gyrus in the hemisphere examined.

Short-term survival analysis: experiment 2. Determination of BrdU- positive cell numbers was accomplished by using the optical fractionator procedure for design-based stereology (West et al., 1991; Peterson, 1999). Data for experiment 2 were collected from immunoperoxidase-stained sections with an Olympus BX51 equipped with $10 \times$ and $60 \times$ (oil; 1.4 NA) lenses, a motorized $\mathrm{x}-\mathrm{y}-\mathrm{z}$ stage control, and the StereoInvestigator software (MBF Bioscience). The stereological parameters for experiment 1 were as follows: sampling grid, $100 \times 100 \mu \mathrm{m}$; counting frame, $75 \times 75 \mu \mathrm{m}$. Section thickness was measured directly at each site with the optical dissector counting frame height set at $20 \mu \mathrm{m}$, which is $\sim 70 \%$ of mounted section thickness with this type of histological processing.

Differentiation-long-term survival analysis: experiment 3. To detect BrdU coexpression with markers of lineage commitment, sections were stained for multiple immunofluorescence. Stereological sampling was performed with a confocal laser scanning microscope (Olympus Fluoview 300) for determination of multiple phenotype markers coexpression in BrdU-positive cells, as described above, with $10 \times$ and $100 \times$ (oil; $1.0 \mathrm{NA}$ ) lenses. The following excitation wavelengths were used: $488 \mathrm{~nm}$ for the secondary fluorophore Cy2 (BrdU), 568 $\mathrm{nm}$ for the secondary fluorophore $\mathrm{Cy} 3$ $(\mathrm{NeuN})$, and $647 \mathrm{~nm}$ for the secondary fluorophore Cy5 $(\mathrm{S} 100 \beta)$. Systematic random sampling was achieved as described above for counting immunoperoxidase-stained sections. The stereological parameters used for experiment 3 were as follows: sampling grid, $200 \times 200 \mu \mathrm{m}$; counting frame, $100 \times 100 \mu \mathrm{m}$. Section thickness was measured directly at each site with the optical dissector counting frame height set at $30 \mu \mathrm{m}$, which is $\sim 70 \%$ of mounted section thickness with this type of histological processing. Colocalization was determined by coexpression of separately collected emission channels examined at multiple focal planes through each cell. This procedure sampled at least $5 \%$ of the total hippocampal dentate gyrus in the hemisphere examined.

\section{Statistical analysis}

Unpaired Student's $t$ tests were used to evaluate group mean differences between control and stressed animals for comparison of cell number estimates determined from stereological sampling and for CORT level differences. Results are expressed as the mean \pm SEM. Significance for all unpaired Student's $t$ tests was set at a level of $p<0.05$. Pearson $r$ correlation coefficient was calculated to determine whether a correlation existed between CORT levels and BrdU-positive cell numbers and between bites and BrdU-positive cell numbers for experiment 2 intruder subjects. GB-STAT (Dynamic Microsystems, Silver Spring, MD) was used for all statistical analyses.

\section{Results \\ Response to psychosocial stress}

Behavior

As expected, intruder and resident rats readily engaged in competition over territory and established a clear dominant (resident rat) and subordinate (intruder rat) relationship during the 20 min defeat session. During this time, the subordinate intruder rat displayed specific stress-induced subordinate behavior, including submissive supine posturing, freezing, and flight-avoidance. All intruder rats vocalized, exhibited submissive posture, and were pinned down by a resident rat within $45 \mathrm{~s}$ of introduction into the colony, thus meeting criteria (Fig. 2). Additionally, twothirds of the intruder rats received bites from the resident rats, and $66 \%$ of those bitten received five or more bites. Thus all 
intruder animals received stressors above the established criteria and exhibited behavior indicative of stress.

\section{CORT levels}

Elevation of plasma CORT is one indication of biological response to stress. To confirm the presence of stress after the psychosocial stress episode, plasma CORT levels were analyzed for experiment 2 subjects. A concomitant elevation of plasma CORT levels generally occurs in subordinate animals (Zelena et al., 1999; Kozorovitskiy and Gould, 2004). In our experiment, plasma CORT levels were elevated more than six-fold in animals exposed to psychosocial stress. A measurement of serum CORT levels immediately after either a sham cage session for control animals or a stress episode for intruder rats showed significantly increased CORT levels in rats experiencing the psychosocial stress episode. The mean CORT measure for control animals was $23.25 \pm 8.31$ $\mathrm{ng} / \mathrm{ml}$, whereas the CORT level mean for the intruder animal was $147 \pm 40.1 \mathrm{ng} / \mathrm{ml}$. Therefore, intruder subjects exhibited a significant $(p \leq 0.01)$ increase in the level of CORT that was indicative of stress. Both the elevated CORT levels and the behavioral changes from this single episode of psychosocial stress may be long lasting.

Increased CORT has been proposed to be a direct mechanism for diminished neurogenesis; however, the literature is mixed in support of this conclusion (Gould et al., 1998). One explanation for discrepancies in the literature may be temporal limitations on the activation of mineralocorticoid and glucocorticoid receptors during the stages of neurogenesis (Cameron and McKay, 1999; Montaron et al., 2003; Garcia et al., 2004; Heine et al., 2004). Glucocorticoid and mineralocorticoid receptors are not expressed, or of restricted expression, during the initial proliferation phase, suggesting that CORT elevation may impact subsequent short- and long-term survival phases of neurogenesis but not directly regulate proliferation or immediate survival (Garcia et al., 2004). This interpretation of the delayed impact of alteration of CORT levels is further substantiated by the subsequent results of the present study, in which elevated CORT levels exist without a corresponding reduction in cell proliferation or immediate survival.

\section{Psychosocial stress exposure does not alter proliferation}

Experiment 1 subjects in intruder and control groups were examined for newly generated or proliferating cells in the dentate granule cell layer at the time of exposure to psychosocial stress (Fig. $1 B)$. Both groups showed similar populations of CldU-positive cells in the subgranular zone of the hippocampus (Fig. 3C,D), indicating that exposure to psychosocial stress did not change the number of proliferating cells in the dentate gyrus. Stereological estimates were performed to assess the number of newly generated CldU-positive cells. Stereological estimates of newly generated cells in the dentate gyrus and subgranular zone of intruder animals were $2898 \pm 391$ compared with control animals at $1800 \pm 265$. With a $p$ value of 0.068 , there was no statistical difference in the proliferation rate between these two groups.

\section{Psychosocial stress exposure does not alter the immediate survival of newly generated cells}

To discriminate the effect of psychosocial stress on the immediate survival of newly generated cells separate from its effect on their generation, subjects experienced psychosocial stress $1 \mathrm{~d}$ after cells were labeled with IdU, and the immediate survival of IdU-labeled cells was evaluated (Fig. $1 \mathrm{~B}$ ). IdU-positive cells within the dentate gyrus were detected in both control and intruder animals (Fig.
$3 E, F)$. Stereological estimates of the number of IdU-positive cells surviving at $2 \mathrm{~d}$ in control animals $(4041 \pm 373)$ and intruder animals $(5184 \pm 726)$ did not differ $(p=0.23)$. Thus, an acute psychosocial stressor did not affect the immediate survival of newly generated cells.

\section{Reentry of newly generated cells into the cell cycle is unaltered}

In addition to individual CldU and IdU cell labeling, a portion of cells expressed both cell markers (Fig. $3 G, H$ ). This population is defined as those cells cycling during the presence of CldU that had also gone through S-phase in the previous $48 \mathrm{~h}$ when IdU was present. Therefore, this number is an indication of population cell cycle activity based on the distribution of IdU, CldU, and colocalized cells for both control and intruder animals. The mean percentage of recycling cells (coexpressing CldU and IdU) was $24.6 \pm 1.9 \%$ for control animals and $26.25 \pm 7.4 \%$ for intruders $(p=0.83)$. This suggests that stress does not alter the frequency of the cell cycle in the population of cells already engaged in DNA replication.

To address the phenotypic identity of the proliferating cells, a series of sections from animals in experiment 1 were stained for coexpression of IdU and CldU with DCX, a marker of early neuronal lineage commitment (Fig. 3I-L). Every sixth section through the hippocampus was imaged, and sequential confocal images stacks were obtained throughout the section thickness. Because the purpose of this analysis was to estimate the phenotypic distribution of proliferating cells, the selection of sampling sites was weighted to the presence of proliferating cells rather than following strict stereological systematic random sampling. At each site, the coexpression of IdU, CldU, and DCX was assessed in three dimensions with the StereoInvestigator software (MBF Bioscience) to record the phenotypic scoring.

In agreement with the stereological data on dual thymidinelabeled cells, there was no significant overall difference between control and intruder animals in regard to DCX expression. In control animals, $71.4 \pm 4.0 \%$ of cycling cells coexpressed DCX, whereas this population was slightly elevated in intruder animals to $79.4 \pm 4.2 \%$. The coexpression with DCX indicates that those cycling cells are a transit-amplifying population in an early period of neuronal lineage commitment. Similarly, in both conditions, about one-fourth of the cycling cells did not coexpress DCX, suggesting that this portion of the population is earlier in lineage commitment or even uncommitted, because this staining pattern may identify slowly cycling stem cells.

Proliferating cells were also scored on the basis of their combination of halogenated thymidine analog expression (IdU positive only, CldU positive only, or dual expression of IdU and CldU) (Fig. 3I-L) along with the presence or absence of DCX expression. When this individual thymidine analog subpopulation staining was assessed, there was a difference in the coexpression of IdU-positive cells (those labeled before stress and representing the population addressing the immediate survival of newly generated cells) in regard to DCX coexpression. IdU+/ CldU - cells in intruder animals coexpressed DCX in $41.9 \pm$ $1.8 \%$ of the population, compared with only $26.3 \pm 6.3 \%$ in control animals. These data reflect a shift to an increased percentage of IdU $+/ \mathrm{CldU}$ - cells with neuronal commitment under stress conditions, despite there being no change in the overall number of cycling cells. This phenotypic shift to increased frequency of coexpression with DCX may reflect an increased selective pressure to neuronal lineage commitment in response to the experience of psychosocial stress. 


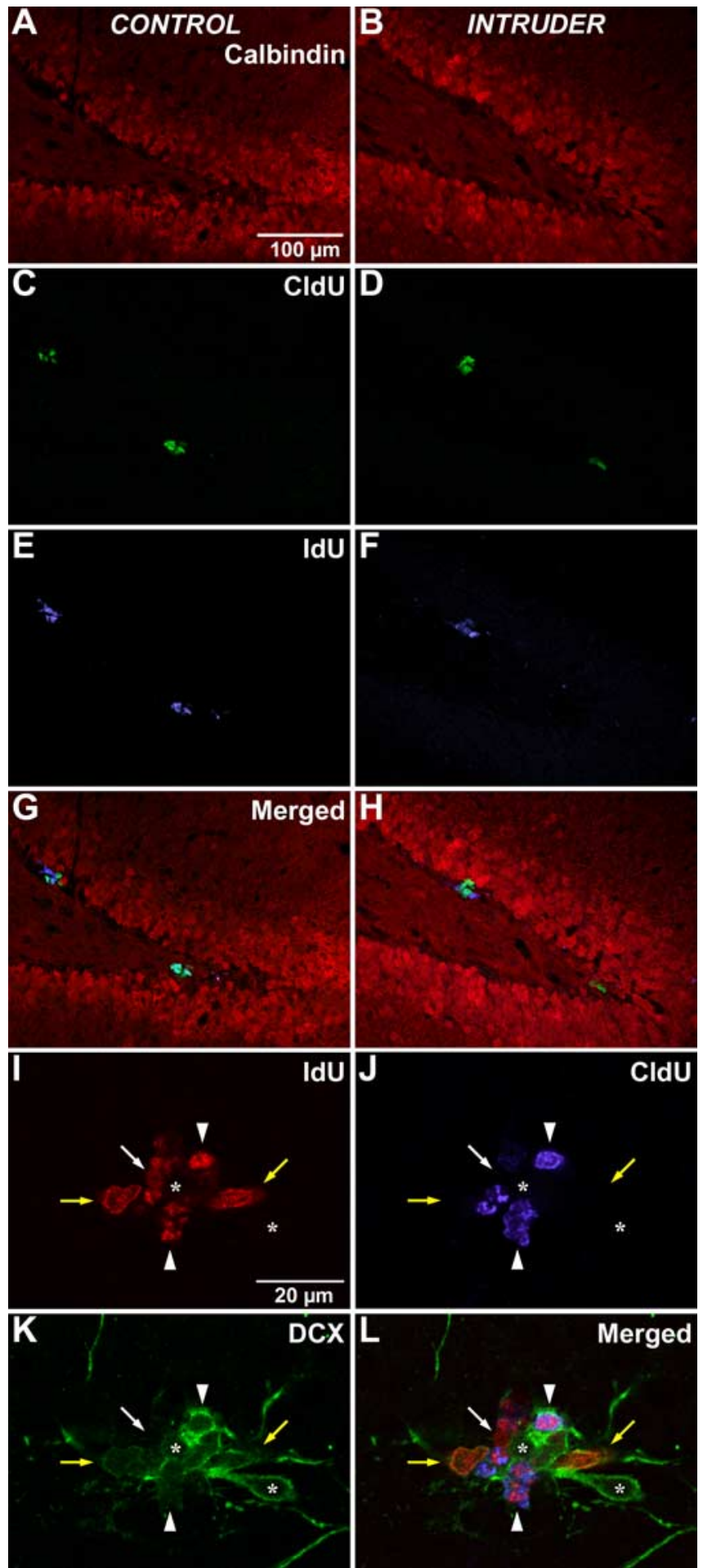

Figure 3. Immunohistochemical detection of halogenated thymidine analogs. Animals that had been administered IdU for $2 \mathrm{~d}$ followed by $\mathrm{CldU}$ coincident with experiencing the episode of stress were evaluated $1 \mathrm{~d}$ later (Fig. $1 B$, experiment 1). $\boldsymbol{A}, \boldsymbol{B}$, Sections from control $(\boldsymbol{A})$ or intruder $(\boldsymbol{B})$ rats were stained with calbindin (red) to identify the granule cell layer of the dentate gyrus. $\boldsymbol{C}, \boldsymbol{D}$, Detection of CldU (green) identified cells that were undergoing DNA replication on the day of the psychosocial stress episode. $\boldsymbol{E}, \boldsymbol{F}$, Detection of IdU (blue) identified cells that had been generated immediately before the stress episode. Examination of the merged image permits additional detection of cells that coexpress both IdU and CIdU, indicating cells that have reentered the cell cycle during the presence of these two thymidine analogs. There is no apparent difference between control rats $(\boldsymbol{A}, \boldsymbol{C}, \boldsymbol{E}, \boldsymbol{G})$ and intruder rats $(\boldsymbol{B}, \boldsymbol{D}, \boldsymbol{F}, \boldsymbol{H})$. Scale bar: (in $\boldsymbol{A}) \boldsymbol{A}-\boldsymbol{H}, 100 \mu \mathrm{m}$. In addition, coexpression of IdU ( $\boldsymbol{I}$, red) and CldU ( $\boldsymbol{J}$, blue) with DCX ( $\boldsymbol{K}$, green) in the subgranular zone of the dentate gyrus was assessed to evaluate the phenotypic distribution of newly generated cells with this marker of early neuronal linage commitment. $\boldsymbol{L}$,
Although three-fourths of proliferative cells coexpressed DCX in experiment 1 , most DCX cells in the dentate gyrus were not proliferating. Coexpression with thymidine analogs was found in $<20 \%$ of DCX-positive cells in the dentate gyrus, indicating that most of the DCX-positive cell population are either postmitotic or have a cycling frequency of $>3 \mathrm{~d}$. Coexpression with thymidine analogs was typically noted in the DCX-positive cells in the basal portion of the subgranular zone, with morphological polarity running parallel to the base of the granule cell layer. This observation is consistent with previous descriptions of this population as more primitive, in contrast to cells with apical process extension into the granule cell layer that have been described as more mature DCXpositive cells (Kempermann et al., 2004).

\section{Psychosocial stress exposure reduces short-term survival of} newly generated cells

Because there was no alteration in proliferation or immediate survival, a second experiment examined the subsequent survival of these newly generated cells. Subjects in experiment 2 were examined for short-term survival (up to 1 week) of cells that were newly generated at the time of exposure to psychosocial stress (Fig. 1C). Control animals had a robust population of BrdUpositive cells in the subgranular zone of the hippocampus (Fig. $4 A)$. In contrast, the intruder animals had fewer BrdU-positive cells in the same region (Fig. $4 B$ ).

To quantify differences in these populations between experimental and control groups, stereology was used to estimate the number of newly generated BrdU-positive cells. Exposure of the animals to BrdU over a period of $72 \mathrm{~h}$ labels a cohort of cells proliferating during this period. Estimation of BrdU + cell populations in the hippocampus of control animals revealed that $\sim 3900$ cells were generated per day and survived until the time of examination 1 week later. Intruder animals that received a psychosocial stress concurrent with the time of BrdU exposure showed a significant reduction of BrdU-labeled cells to approximately one-third of control levels, or $\sim 1200$ cells per day $(p=0.021)$. The expression of results as a daily value was based on the estimates of the total population of cells over $72 \mathrm{~h}$ of BrdU exposure (control, $11730 \pm 1131$; intruder, $3705 \pm 390$ ) normalized to a $1 \mathrm{~d}$ value. Values are expressed as daily rates to facilitate comparison with other published studies (Kempermann and Kronenberg, 2003). All intruder subjects in experiment 2 exhibited diminished BrdU-labeled cells; however, there was no correlation between BrdU cell number and the number of bites or CORT levels, indicating that there was not a threshold effect or gradation in severity for stress to affect cell number. All animals in experiment 2 exhibited significantly diminished shortterm cell survival regardless of the amount of CORT increase from baseline or the number of bites received. These data did not reveal a dose-response with an acute episode of psychosocial stress. It is possible that a dose-response exists between stress and neurogenesis under certain stress paradigms, but it

\section{$\leftarrow$}

A single focal plane from a sequential series of confocal micrographs from an intruder animal is shown merged. The separation of fluorescence emission in $\boldsymbol{I}-\boldsymbol{K}$ reveals that some IdU-positive cells coexpress DCX (yellow arrows) and some do not (white arrow). Some IdU-positive cells also incorporate CldU (arrowheads), indicating that they had just reentered the cell cycle. In this example, the IdU $+/ C l d U+$ cells coexpress DCX, although dual-labeled cells and IdU $-/ C l d U+$ cells can be observed that do not coexpress DCX (data not shown). Not all DCX-positive cells in the subgranular zone are engaged in DNA replication (asterisks). Scale bar: (in $I$ ) $I-L, 20 \mu \mathrm{m}$. 
is apparent that the stress of an encounter such as the psychosocial stress used in this study is of sufficient magnitude and direction to have only detrimental effects.

\section{Psychosocial stress exposure after cell proliferation reduces long-term survival of newly generated cells but not frequency of differentiation}

To further elucidate the role of stress on cell survival, a third group (experiment 3 ) (Fig. 1D) experienced psychosocial stress 1 week after cells were labeled with BrdU, which permitted long-term survival and differentiation of BrdU-labeled cells to be evaluated. These data were obtained with triple immunofluorescence labeling to discriminate between BrdU-positive cells coexpressing phenotypic markers for neurons or astrocytes.

BrdU-positive cells, neurons, and astrocytes within the dentate gyrus were detected in both control and experimental conditions (Fig. 5). The number of BrdUpositive cells normalized on a per day basis surviving at 4 weeks in control animals was $2160 \pm 178$, whereas intruder animals had $1596 \pm 106$ BrdU-positive cells (total for control, $6480 \pm 218$; total for intruder, $4788 \pm 110$ ). This $26 \%$ reduction in BrdU cell number was statistically significant $(p=0.025)$. Therefore, psychosocial stress exposure after cell proliferation reduced the long-term survival of newly generated cells.

The phenotype of the surviving BrdUpositive cells was examined and counted three-dimensionally by using stereological sampling to determine coexpression of mature neuronal or astrocytic markers. Evaluation of the neuronal differentiation of these cells showed that the number of BrdU/ NeuN-colabeled cells surviving at 4 weeks in control animals, normalized on a per day basis, was $1225 \pm 135$; in intruder rats, it was $820 \pm 121$ (total for control, $3675 \pm 165$; total for intruder, $2460 \pm 121$ ). This $33 \%$ decrease of cells that had differentiated into neurons 4 weeks after the psychosocial stress encounter and cell generation was statistically significant $(p=0.046)$, indicating an overall reduction of new neurons 4 weeks after psychosocial stress exposure. The number of BrdU/S100 $\beta$-colabeled cells per day was negligible (less than one detected per animal on average) for both groups, indicating that few proliferating cells differentiated into astrocytes.

An analysis of the percentage of NeuN cells colocalized with BrdU revealed that $56.4 \pm 3 \%$ of BrdU-positive cells also expressed NeuN in control animals and $49.5 \pm 5 \%$ of BrdUpositive cells expressed NeuN in experimental animals. This difference was not statistically significant $(p=0.226)$. The fact that the percentage of BrdU-positive cells coexpressing a neuronal marker, NeuN, is the same for both experimental and control groups indicates that psychosocial stress after cell proliferation did not impede differentiation but did diminish cell survival (and thus the ultimate number of newly differentiated neurons).

\section{Discussion}

We report that an acute episode of psychosocial stress diminishes both short-term survival of proliferating cells and longterm survival of newly differentiated neurons in the dentate gyrus of the hippocampus (experiments 2 and 3 ). The percentage of newly generated cells that differentiated into neurons, however, was not changed with acute psychosocial stress, indicating that the stress episode did not affect the process of differentiation of newly generated cells but rather affected their survival (experiment 3). An alternative interpretation is that stress impeded cellular differentiation and lineage arrest resulted in cell death. Furthermore, acute psychosocial stress did not reduce either proliferation or immediate survival of newly generated cells in the dentate gyrus of subjects at the 1 and $2 \mathrm{~d}$ survival time points, despite endocrine and behavioral responses of intruder rats that were indicative of stress (experiment 1). The lack of difference in the proportion of cells reentering the cell cycle between control and stressed animals is another indicator that stress was not affecting proliferation. These results suggest that the stress-related reduction in the population of newly generated cells occurred after their generation. 

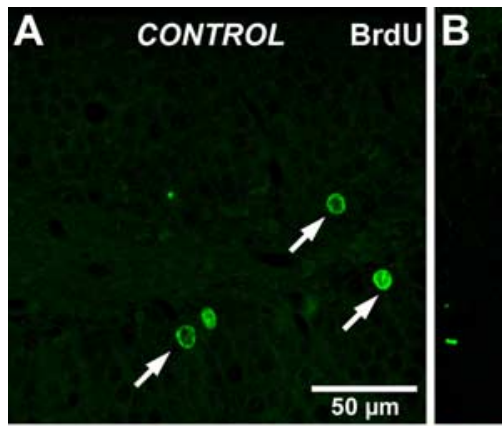

INTRUDER

BrdU
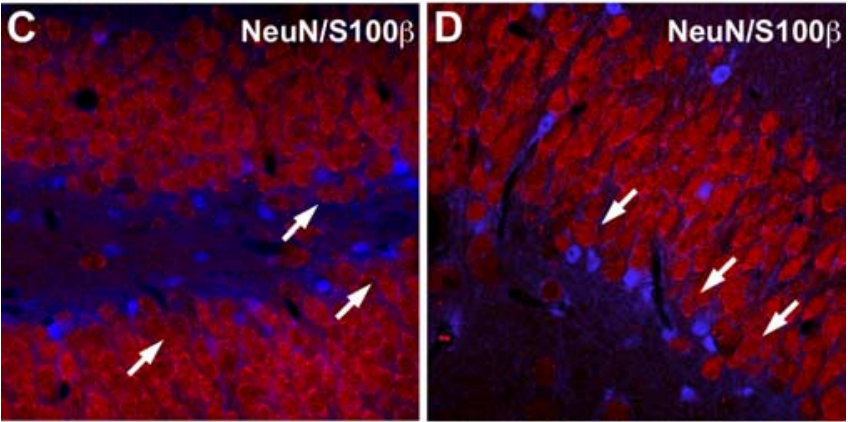

$\mathbf{E}$
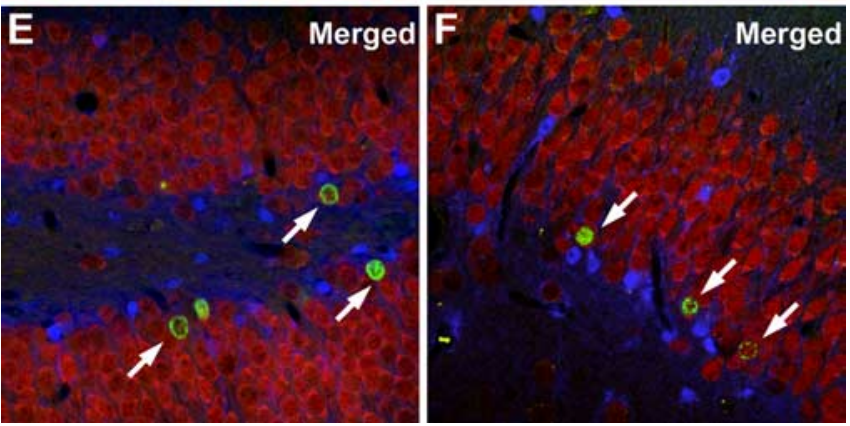

Figure 5. Neuronal differentiation of newly generated cells. Shown is the immunohistochemical detection of BrdU-positive cells in animals that had received BrdU 1 week before experiencing the single episode of psychosocial stress and that were examined at 4 weeks to evaluate their long-term survival (Fig. 1D, experiment 3). $\boldsymbol{A}, \boldsymbol{B}$, Cells generated during the delivery of BrdU (green) before the stress episode could be observed in the dentate gyrus of both control and intruder animals. $\boldsymbol{C}-\boldsymbol{F}$, In both cases, the BrdU-positive cells frequently coexpressed the mature neuronal marker NeuN (red), but only very rarely was any colocalization observed with the mature astrocytic marker $\$ 100 \beta$ (blue). Colocalization was determined on the basis of three-dimensional analysis of coexpression within the same cell imaged by using sequential focal planes obtained by confocal microscopy. Scale bar: (in $\boldsymbol{A}) \boldsymbol{A}-\boldsymbol{F}, 50 \mu \mathrm{m}$.

\section{Neurogenic proliferation is unaffected by psychosocial stress}

Using sequential administration of dual thymidine analogs IdU and CldU (Vega and Peterson, 2005), we found that acute psychosocial stress did not affect proliferation or immediate survival, despite evidence of diminished short- and long-term survival of newly generated cells. The fact that there was no change in the percentage of cells reentering the cell cycle at the time of stress is another indicator that proliferation is unaffected by the episode of psychosocial stress. The measure of proliferation between 12 and $24 \mathrm{~h}$ after thymidine analog delivery results in coarser resolution of this parameter than if animals had been killed after a shorter interval; however, because the cell cycle of neural progenitor-stem cells in the rodent CNS has been reported to take at least $17 \mathrm{~h}$ (Coskun and Luskin, 2002), it is likely that the CldUpositive cell population in experiment 1 primarily reflects a single episode of proliferation within this interval. In fact, the estimate of dual-labeled CldU/IdU-positive cells shows that reentry to the cell cycle within $1 \mathrm{~d}$ could not exceed $25 \%$ of the proliferation population estimate and is likely much less.
These findings suggest a finer division of neurogenic modulation than may have been considered in the past. Previous studies that reported diminished proliferation may have actually been observing diminished short-term survival dependent on the timing of their BrdU birth dating (Gould et al., 1997, 1998). Oversimplification of the process of neurogenesis by not accounting for progression from proliferation through the stages of lineage commitment in previous studies may explain those inconsistent reports in the literature that have generated controversy about the role of stress. Our data suggest that psychosocial stress effects on neurogenesis are not immediate but may take $>24 \mathrm{~h}$ for changes to be manifested at the cellular level. Therefore, there may be a change in the cellular environment that diminishes cell survival after the proliferative event (because acute stress is not altering proliferation). This finding is consistent with the report of a predator odor that induced stress but failed to diminish proliferation in rats, despite robust biological indications of stress, including elevated CORT levels (Thomas and Peterson, 2006). Thus, elucidating the mechanisms differentially regulating neurogenesis is of importance for discerning the possible relevance of neurogenesis to human clinical depression (Arango et al., 2001; Benninghoff et al., 2002).

\section{Psychosocial stress reduces survival without altering differentiation}

In the present study, a single episode of psychosocial stress induced a robust decrease in short-term survival of newly generated cells in the hippocampus of intruder subjects. This finding is novel in that survival of newly generated cells has not been examined previously in a social defeat paradigm; however, similar effects have been reported in other studies in rodents and higher order mammals by using different stress models. Malberg and Duman (2003) used inescapable shock as an acute stressor, with resultant long-lasting diminished cell numbers. Likewise, chronic psychosocial stress to adult male tree shrews reduced hippocampal cell numbers (Czeh et al., 2002).

The influence of psychosocial stress on regulating the survival of newly generated cells is further illustrated by the results from experiment 3 (Fig. $1 D$ ). Subjects exposed to stress 1 week after a cohort of cells had been "birth dated" by administration of BrdU had reduced survival of those previously generated cells; however, although the total number of new cells was reduced, the percentage of surviving neurons was not different from controls. The long-term survival effect may be a byproduct of the effects of stress on short-term survival affecting the cumulative number of newly differentiated neurons. This finding is in contrast to that of Malberg and Duman (2003), who used a chronic learnedhelplessness paradigm and found a transient decrease in proliferation-immediate survival that was no longer present at 4 weeks. It is possible that the animals became habituated to the chronic restraint stress used in the restraint paradigm, allowing for a compensatory response that diminished its long-term effect on survival. In the present study, diminished short-term survival after a potent, acute psychosocial stress was long-lasting and not overcome by the 4 week time point.

\section{Equilibrium of neurogenesis}

Many factors modulate the process of neurogenesis, indicating that neurogenesis is a dynamic process sensitive to social and environmental cues (Peterson, 2002; Lennington et al., 2003). Although proliferation has typically been investigated, our data indicate that survival is a key factor in the psychosocial stress paradigm. A state of equilibrium may exist between cell birth and 
death, and environmental stimuli such as stress may alter this balance, leading to disruption of homeostasis in the hippocampus (Lennington et al., 2003; Heine et al., 2004). In the present study, the immediate survival group (experiment 1 ) did not show a difference in cell number as compared with control animals, but the short-term survival group (experiment 2 ) did show a reduction in newly generated cells. Thus, a change in cell status apparently occurred in the first week after the new cells had been generated.

The observation that the cell loss in our study occurred at a time interval of $>24 \mathrm{~h}$ suggests that there may have been an alteration of the environmental niche within the dentate gyrus as a result of the experience of psychosocial stress. The fact that CORT levels were elevated without altering the immediate survival of new cells suggests that the stressor effect may not be mediated directly in response to hypothalamic-pituitary-adrenal axis activation (Thomas et al., 2006). The absence of glucocorticoid receptors in newly generated cells (Cameron et al., 1998; Mirescu et al., 2004), or their expression in a small percentage of proliferating cells (Garcia et al., 2004), is consistent with this interpretation. The observation that the number of new cells expressing glucocorticoid receptors increases by $4 \mathrm{~d}$ after their generation (Garcia et al., 2004) indicates that any direct effect of elevated CORT is delayed until after their generation, possibly contributing to reduced cell survival; however, glucocorticoid receptors are present on astrocytes in the hippocampus, suggesting that CORT influence may actually be indirect, secondary to activation of regional astrocytes (Song et al., 2002; Chadashvili and Peterson, 2006). Together, these observations suggest that acute psychosocial stress may impact neurogenesis indirectly by shifting the expression of regulatory factors within the environmental niche of the hippocampus (Wong and Herbert, 2005), with possible direct effects not contributing until several days after proliferation.

\section{Conclusions}

It has recently been proposed that diminished neurogenesis is a key factor in the pathogenesis of depression (Duman et al., 1999, 2001a,b; D'Sa and Duman, 2002; Garcia, 2002; Kempermann, 2002). The rationale for this view is that stress diminishes neurogenesis, and clinical depression is often associated with stress (Jacobs et al., 2000a,b). It has even been concluded that neurogenesis is a "requirement" for certain antidepressant behavioral effects (Santarelli et al., 2003), but it is still unknown whether diminished neurogenesis could be a cause, a consequence, or a correlate of depression (Gage, 2000; Thomas and Peterson, 2003). It is an important finding that an acute episode of psychosocial stress did not alter proliferation or immediate survival, as evidenced by IdU/CldU administration in experiment 1 , but it did alter short- and long-term survival as shown in experiments 2 and 3. Therefore, a single episode of stress may initiate a cascading response within the hippocampal environment that may alter the milieu to make it less supportive of the survival of newly generated cells.

Given the evidence that reduction of neurogenesis may be a contributing factor to depression, the restoration of neurogenesis after stressful episodes may be of potential value for understanding the biological underpinning of clinical depression. Future studies are needed to pinpoint when this alteration of the neurogenic environment occurs. Once this time course has been ascertained, it may be used to define a "therapeutic window" during which exogenous regulatory factors can be effectively introduced to modulate neurogenesis through promoting the survival of newly generated cells.

\section{References}

Arango V, Underwood MD, Boldrini M, Tamir H, Kassir SA, Hsiung S, Chen JJ Mann JJ (2001) Serotonin 1A receptors, serotonin transporter binding and serotonin transporter mRNA expression in the brainstem of depressed suicide victims. Neuropsychopharmacology 25:892-903.

Benninghoff J, Schmitt A, Mossner R, Lesch KP (2002) When cells become depressed: focus on neural stem cells in novel treatment strategies against depression. J Neural Transm 9:947-962.

Blanchard RJ, McKittrick CR, Blanchard DC (2001) Animal models of social stress: effects on behavior and brain neurochemical systems. Physiol Behav 73:261-271.

Cameron HA, McKay RD (1999) Restoring production of hippocampal neurons in old age. Nat Neurosci 2:894-897.

Cameron HA, Hazel TG, McKay RD (1998) Regulation of neurogenesis by growth factors and neurotransmitters. J Neurobiol 36:287-306.

Chadashvili T, Peterson DA (2006) Cytoarchitecture of fibroblast growth factor receptor 2 (FGFR-2) immunoreactivity in astrocytes of neurogenic and non-neurogenic regions of the young adult and aged rat brain. J Comp Neurol 498:1-15.

Coskun V, Luskin MB (2002) Intrinsic and extrinsic regulation of the proliferation and differentiation of cells in the rodent rostral migratory stream. J Neurosci Res 69:795-798.

Czeh B, Michaelis T, Watanabe T, Frahm J, de Biurrun G, van Kampen M, Bartolomucci A, Fuchs E (2001) Stress-induced changes in cerebral metabolites, hippocampal volume, and cell proliferation are prevented by antidepressant treatment with tianeptine. Proc Natl Acad Sci USA 98:12796-12801.

Czeh B, Welt T, Fischer AK, Erhardt A, Schmitt W, Muller MB, Toschi N, Fuchs E, Keck ME (2002) Chronic psychosocial stress and concomitant repetitive transcranial magnetic stimulation: effects on stress hormone levels and adult hippocampal neurogenesis. Biol Psychiatry 52:1057-1065.

D'Sa C, Duman RS (2002) Antidepressants and neuroplasticity. Bipolar Disord 4:183-194.

Duman RS, Malberg J, Thome J (1999) Neural plasticity to stress and antidepressant treatment. Biol Psychiatry 46:1181-1191.

Duman RS, Malberg J, Nakagawa S (2001a) Regulation of adult neurogenesis by psychotropic drugs and stress. J Pharmacol Exp Ther 299:401-407.

Duman RS, Nakagawa S, Malberg J (2001b) Regulation of adult neurogenesis by antidepressant treatment. Neuropsychopharmacology 25:836-844.

Encinas JM, Vashtokari A, Enikolopov G (2006) Fluoxetine targets early progenitor cells in the adult brain. Proc Natl Acad Sci USA 103:8233-8238.

Fuchs E, Flugge G (1998) Stress, glucocorticoids and structural plasticity of the hippocampus. Neurosci Biobehav Rev 23:295-300.

Fuchs E, Flugge G, Ohl F, Lucassen P, Vollmann-Honsdorf GK, Michaelis T (2001) Psychosocial stress, glucocorticoids, and structural alterations in the tree shrew hippocampus. Physiol Behav 73:285-291.

Gage FH (2000) Structural plasticity: cause, result or correlate of depression. Biol Psychiatry 48:713-714.

Gage FH, Coates PW, Palmer TD, Kuhn HG, Fisher LJ, Suhonen JO, Peterson DA, Suhr ST, Ray J (1995) Survival and differentiation of adult neuronal progenitor cell transplanted to the adult brain. Proc Natl Acad Sci USA 92:11879-11883.

Garcia A, Steiner B, Kronenberg G, Bick-Sander A, Kempermann G (2004) Age-dependent expression of glucocorticoid- and mineralocorticoid receptors on neural precursor cell populations in the adult murine hippocampus. Aging Cell 3:363-371.

Garcia R (2002) Stress, metaplasticity, and antidepressants. Curr Mol Med 2:629-638.

Gould E, Gross CG (2002) Neurogenesis in adult mammals: some progress and problems. J Neurosci 22:619-623.

Gould E, Tanapat P (1999) Stress and hippocampal neurogenesis. Biol Psychiatry 46:1472-1479.

Gould E, McEwen BS, Tanapat P, Galea LA, Fuchs E (1997) Neurogenesis in the dentate gyrus of the adult tree shrew is regulated by psychosocial stress and NMDA receptor activation. J Neurosci 17:2492-2498.

Gould E, Tanapat P, McEwen BS, Flugge G, Fuchs E (1998) Proliferation of granule cell precursors in the dentate gyrus of adult monkeys is diminished by stress. Proc Natl Acad Sci USA 95:3168-3171. 
Gould E, Tanapat P, Rydel T, Hastings N (2000) Regulation of hippocampal neurogenesis in adulthood. Biol Psychiatry 48:715-720.

Heine VM, Maslam S, Zareno J, Joels M, Lucassen PJ (2004) Suppressed proliferation and apoptotic changes in the rat dentate gyrus after acute and chronic stress are reversible. Eur J Neurosci 19:131-144.

Hotsenpiller G, Williams JL (1996) Conditioned fear and analgesia to conspecific odors: benzodiazepine and 5-HT[1A] agonists. Psychobiology 24:118-126.

Jacobs BL, van Praag H, Gage FH (2000a) Adult brain neurogenesis and psychiatry: a novel theory of depression. Mol Psychiatry 5:262-269.

Jacobs BL, van Praag H, Gage FH (2000b) Depression and the birth and death of brain cells. Am Sci 88:340-345.

Kempermann G (2002) Regulation of adult hippocampal neurogenesis: implications for novel theories of major depression. Bipolar Disord 4:17-33.

Kempermann G, Kronenberg G (2003) Depressed new neurons: adult hippocampal neurogenesis and a cellular plasticity hypothesis of major depression. Biol Psychiatry 54:499-503.

Kempermann G, Jessberger S, Steiner B, Kronenberg G (2004) Milestones of neuronal development in the adult hippocampus. Trends Neurosci 27:447-452.

Kessler RC (1997) The effects of stressful life events on depression. Annu Rev Psychol 48:191-214.

Kornack DR, Rakic P (1999) Continuation of neurogenesis in the hippocampus of the adult macaque monkey. Proc Natl Acad Sci USA 96:5768-5773.

Kozorovitskiy Y, Gould E (2004) Dominance hierarchy influences adult neurogenesis in the dentate gyrus. J Neurosci 24:6755-6759.

Lennington JB, Yang Z, Conover JC (2003) Neural stem cells and the regulation of adult neurogenesis. Reprod Biol Endocrinol 1:99.

Malberg JE (2004) Implications of adult hippocampal neurogenesis in antidepressant action. J Psychiatry Neurosci 29:196-205.

Malberg JE, Duman RS (2003) Cell proliferation in adult hippocampus is decreased by inescapable stress: reversal by fluoxetine treatment. Neuropsychopharmacology 28:1562-1571.

McEwen BS (2000) The neurobiology of stress: from serendipity to clinical relevance. Brain Res 886:172-189.

Meerlo P, Koehl M, van der Borght K, Turek FW (2002) Sleep restriction alters the hypothalamic-pituitary-adrenal response to stress. J Neuroendocrinol 14:397-402.

Mirescu C, Peters JD, Gould E (2004) Early life experience alters response of adult neurogenesis to stress. Nat Neurosci 7:841-846.

Montaron MR, Piazza PV, Aurousseau A, Urani ML, Le Moal M, Abrous DN
(2003) Implication of corticosteroid receptors in the regulation of hippocampal structural plasticity. Eur J Neurosci 18:3105-3111.

Peterson DA (1999) Quantitative histology using confocal microscopy: implementation of unbiased stereology procedures. Methods 18:493-507.

Peterson DA (2002) Stem cells in brain plasticity and repair. Curr Opin Pharmacol 2:34-42.

Peterson DA (2004) The use of fluorescent probes in cell counting procedures. In: Quantitative methods in neuroscience (Evans S, Jansen AM, Nyengaard JR, eds), pp 85-114. New York: Oxford UP.

Pham K, Nacher J, Hof PR, McEwen BS (2003) Repeated restraint stress suppresses neurogenesis and induces biphasic PSA-NCAM expression in the adult rat dentate gyrus. Eur J Neurosci 17:879-886.

Santarelli L, Saxe M, Gross C, Surget A, Battaglia F, Dulawa S, Weisstaub N, Lee J, Duman R, Arancio O, Belzung C, Hen R (2003) Requirement of hippocampal neurogenesis for the behavioral effects of antidepressants. Science 301:805-809.

Sheline YI (2000) 3D MRI studies of neuroanatomic changes in unipolar major depression: the role of stress and medical comorbidity. Biol Psychiatry $48: 791-800$.

Song H, Stevens CF, Gage FH (2002) Astroglia induce neurogenesis from adult neural stem cells. Nature 417:39-44

Thomas RM, Peterson DA (2003) A neurogenic theory of depression gains momentum. Mol Interv 3:441-444.

Thomas RM, Peterson DA (2006) Acute exposure to predator odor elicits a robust increase in corticosterone and a decrease in activity without altering proliferation in the adult rat hippocampus. Exp Neurol 201:308-315.

Vega C, Peterson DA (2005) Stem cell proliferative history in tissue revealed by temporal halogenated thymidine analog discrimination. Nat Methods 2:167-169.

West MJ, Slomianka L, Gundersen HJ (1991) Unbiased stereological estimation of the total number of neurons in the subdivisions of the rat hippocampus using the optical fractionator. Anat Rec 231:482-497.

Williams JL, Rogers AG, Adler AP (1990) Prolonged exposure to conspecific and predator odors reduces fear reactions to these odors during subsequent prod-shock tests. Anim Learn Behav 18:453-461.

Wong EY, Herbert J (2005) Roles of mineralocorticoid and glucocorticoid receptors in the regulation of progenitor proliferation in the adult hippocampus. Eur J Neurosci 22:785-792.

Zelena D, Haller J, Halasz J, Makara GB (1999) Social stress of variable intensity: physiological and behavioral consequences. Brain Res Bull 48: 297-302. 